\title{
Algorithm for point-to-point correlation of geometrically nearly similar microscopic objects
}

\author{
L. Siklós* and U. Kuhnt \\ Max-Planck-Institut für biophysikalische Chemie, Abteilung Neurobiologie, Göttingen, F.R.G.
}

\begin{abstract}
An algorithm is presented that compares two quasi similar images by correlating selected points on them - assuming their coordinates are available. The procedure involves translational, magnificational and rotational operations to find corresponding point pairs on the pictures. The algorithm automatically compensates for slight dissimilarities between images and constructs a reference point database for correlation during the evaluation process. Establishment of the reference point networks on the images prior to the examination is avoided.
\end{abstract}

Image correlation; Selected image points; Transformation operation

\section{Introduction}

The wide range of microscopic applications (light microscopy, transmission electron microscopy, scanning electron microscopy, X-ray microanalytical techniques, secondary ion mass spectrometry, etc.) frequently necessitates correlation of diverse images of the same material. Such correlations in material sciences might require the coupling of analytical information with a structural description of the sample [1], or in diagnostic pathology the reliability of a diagnosis might be increased by examining the same cytologic material by different techniques [2-5]. Such comparisons are often performed manually, based on a visual oneto-one correlation of micrographs [6], and are therefore subjective and, in certain applications, inadequate. Computerized image analysis, however, is a promising means for overcoming these

Correspondence: L. Siklós, Max-Planck-Institut für biophysikalische Chemie, Abteilung Neurobiologie, Postfach 2841 D-3400 Göttingen, F.R.G. limitations [7]. Printed images can be captured and directly compared on the screen of the system, assisted by the application software.

Another field where correlative examination of different microscopic pictures is a frequent procedure is the quantitative analysis of three-dimensional structures $[8,9]$. Here, for the unbiased determination of particle numbers by the disector method [10], a comparison of the same field on two sections of the sample obtained at a given distance is an essential requirement.

\section{Description of the procedure}

In the following a numeric procedure is described which is ideally suited for computer-aided systems that manipulate picture coordinates. The algorithm was developed to facilitate the comparative examination of two pictures by prediction of corresponding coordinate pairs on them during the continuous examination of a selected one. It permits, for example, a correlative description of consecutive sections in the electron microscope if 

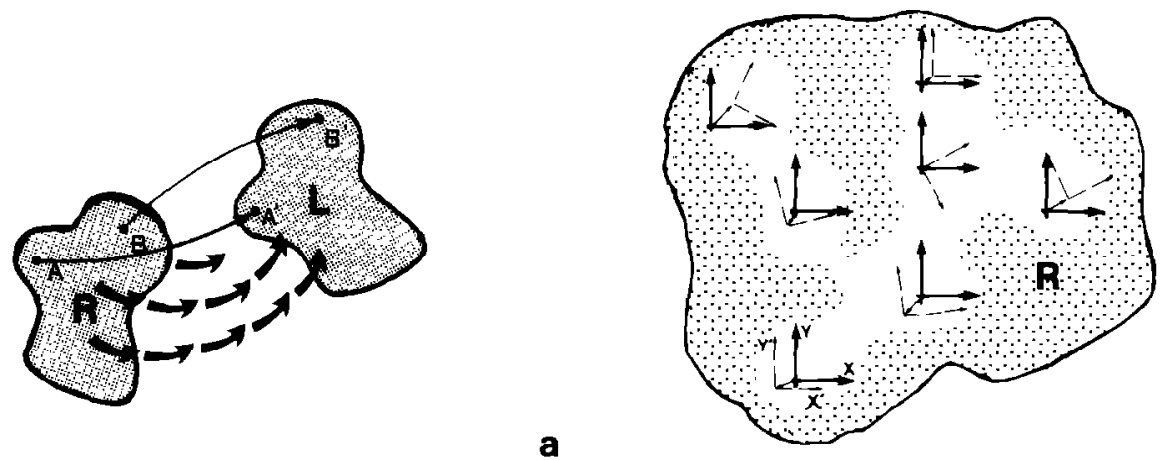

a
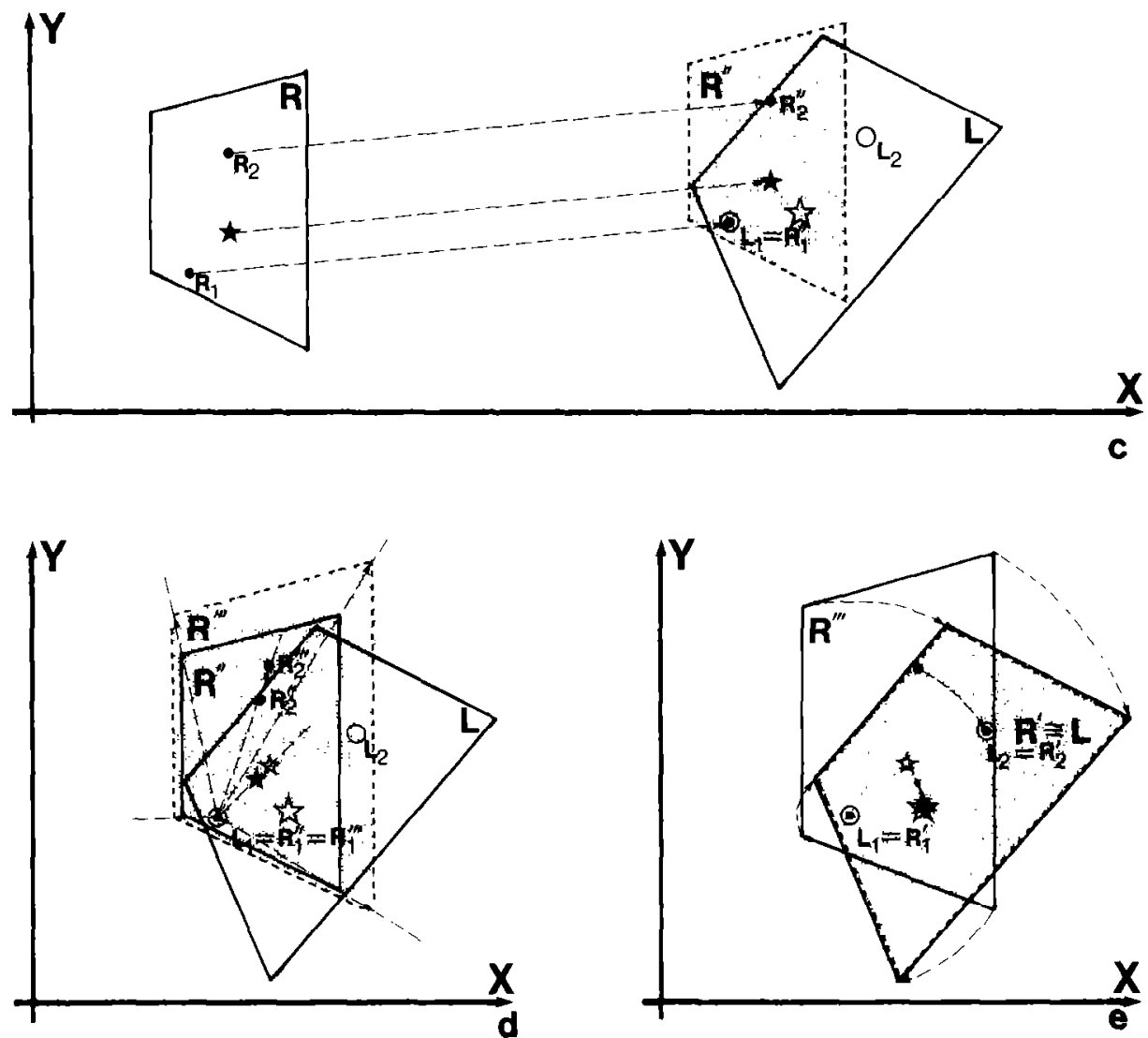

Fig. 1. Principle of point-to-point correlation procedure. (a) Instead of complete superposition of reference (R) and look up (L) objects (thick arrows), correlation of selected points $\left(A \rightarrow A^{\prime}, B \rightarrow B^{\prime}\right.$ ) is aimed. (b) If the objects to be correlated are not completely identical, the necessary translation, rotation and magnification operation is the function of the actual position on the reference object. This space-dependent transformation operation is illustrated by the corresponding reference (solid lines) and look up (dashed lines) coordinate systems plotted for different reference points. (c) The translation operation, illustrated in a Cartesian coordinate system, results in shifted points denoted by $\langle "\rangle, R_{1}, R_{2}$ and $L_{1}, L_{2}$ denote the corresponding reference and look up point pairs which serve to determine the local transformation. The black star denotes the actual point of interest to be compared with a corresponding look up point of unknown position (outlined star). (d) Magnification operation performed on 〈"〉 coordinates results in $\langle " \prime\rangle$ coordinates. (e) Rotation operation $\left\langle^{\prime \prime}\right\rangle$ system results in the final $\left\langle{ }^{\prime}\right\rangle$ transformed coordinates that predict the expected coordinates of the look up point corresponding to our reference point. 
the current coordinates can be read out [11], or more generally, a parallel processing of images stored in automatic image analyser systems after implementing the algorithm as a user's macro. Any small computer-based evaluation system can utilize the algorithm where manipulation (at least reading and storage) of selected picture coordinates is feasible. The advantage of this procedure is obvious when the measures of the details to be correlated are small compared to the specimen size.

Adapting ourselves to a number of applications where the correlation of selected picture points is required, instead of superpositioning complete images (Fig. 1a), our procedure is based on determination only of positions of interest on the two pictures. In practice this means, that during examination of one of them (the reference image - adopting the nomenclature of stereology), when a point of interest is to be correlated with the same field of the other picture (the look-up image), the corresponding picture coordinates have to be predicted. If the images are equivalent or at least geometrically similar, a single transformation formula, determincd once, would apply throughout the study. Under such circumstances the calculation rules of affine transforms would apply without any modification or restriction (see, for example, Refs. 12, 13). However, the geometrical similarity assumption is not generally valid for images in their entirety which were obtained by different methods, because they exhibit different degrees of distortion and astigmatism. Furthermore, images may be inherently slightly different, due to unidentical positions of photography (as in the case of consecutive sections). In such cases the transformation needed to reach a point on the look up picture is the function of the actual position on the reference image, and has to be determined for each point separately (Fig. lb).

In general, superpositioning two similar geometrical objects requires translational, magnificational and rotational operations, which can be calculated exactly from the coordinates of two corresponding point pairs on the reference and look-up images by means of elementary geometrical principles. Given a reference point pair
( $R_{1}(x, y), R_{2}(x, y)$ ) and the corresponding lookup pair $\left(L_{1}(x, y), L_{2}(x, y)\right)$, the necessary calculations to determine $S^{\prime}(x, y)$ on the look-up picture (the corresponding point of $S(x, y)$, our current point of interest on the reference image) are based on the following formulae (overlining denotes section lengths):

Translation by $\overline{R_{1} L_{1}}$ (Fig. 1c):

$$
\begin{aligned}
& \left.\begin{array}{l}
R_{1}^{\prime \prime}(x)=L_{1}(x) \\
R_{1}^{\prime \prime}(y)=L_{1}(y)
\end{array}\right\} \\
& \left.\begin{array}{l}
R_{2}^{\prime \prime}(x)=R_{2}(x)+L_{1}(x)-R_{1}(x) \\
R_{2}^{\prime \prime}(y)=R_{2}(y)+L_{1}(y)-R_{1}(y)
\end{array}\right\} \\
& \left.\begin{array}{l}
S^{\prime \prime}(x)=S(x)+L_{1}(x)-R_{1}(x) \\
S^{\prime \prime}(y)=S(y)+L_{1}(y)-R_{1}(y)
\end{array}\right\}
\end{aligned}
$$

Magnification by factor $M=\overline{L_{2} L_{1}} / \overline{R_{2}^{\prime \prime} R_{1}^{\prime \prime}}$ (Fig. 1d):

$$
\begin{aligned}
& \left.\begin{array}{l}
R_{1}^{\prime \prime \prime}(x)=L_{1}(x) \\
R_{1}^{\prime \prime \prime}(y)=L_{1}(y)
\end{array}\right\} \\
& \left.\begin{array}{l}
R_{2}^{\prime \prime \prime}(x)=L_{1}(x)+M *\left(R_{2}(x)-R_{1}(x)\right) \\
R_{2}^{\prime \prime \prime}(y)=L_{1}(y)+M *\left(R_{2}(y)-R_{1}(y)\right)
\end{array}\right\} \\
& \left.\begin{array}{l}
S^{\prime \prime \prime}(x)=L_{1}(x)+M *\left(S(x)-R_{1}(x)\right) \\
S^{\prime \prime \prime}(y)=L_{1}(y)+M *\left(S(y)-R_{1}(y)\right)
\end{array}\right\}
\end{aligned}
$$

where

$$
M=\sqrt{\frac{\left(L_{2}(x)-L_{1}(x)\right)^{2}+\left(L_{2}(y)-L_{1}(y)\right)^{2}}{\left(R_{2}(x)-R_{1}(x)\right)^{2}+\left(R_{2}(y)-R_{1}(y)\right)^{2}}}
$$

Rotation around $L_{1}$ (Fig. le):

Let us denote:

$$
\begin{array}{ll}
\alpha=\text { steepness of } \overline{L_{1} R_{2}^{\prime \prime \prime}} & \delta=\text { steepness of } \overline{L_{1} S^{\prime \prime \prime}} \\
\beta=\text { steepness of } \overline{L_{1} L_{2}} & \gamma=\text { rotation angle }
\end{array}
$$


The coordinates after the final transformation step:

$$
\left.\begin{array}{l}
\left.\left.\begin{array}{l}
R_{1}^{\prime}(x)=L_{1}(x) \\
R_{1}^{\prime}(y)=L_{1}(y)
\end{array}\right\} \quad \begin{array}{l}
R_{2}^{\prime}(x)=L_{2}(x) \\
R_{2}^{\prime}(y)=L_{2}(y)
\end{array}\right\} \\
S^{\prime}(x)=L_{1}(x)+M * R_{1} S \cos (\delta+\gamma) \\
S^{\prime}(y)=L_{1}(y)+M * R_{1} S \sin (\delta+\gamma)
\end{array}\right\}
$$

where

$$
\overline{R_{1} S}=\sqrt{\left(S(x)-R_{1}(x)\right)^{2}+\left(S(y)-R_{1}(y)\right)^{2}}
$$

and the angles are determined implicitly according to:

$$
\begin{aligned}
& \tan \alpha=\frac{R_{2}(y)-R_{1}(y)}{R_{2}(x)-R_{1}(x)} \quad \tan \delta=\frac{S(y)-R_{1}(y)}{S(x)-R_{1}(x)} \\
& \tan \beta=\frac{L_{2}(y)-L_{1}(y)}{L_{2}(x) \quad L_{1}(x)} \quad \gamma=\beta-\alpha
\end{aligned}
$$

From the formulae pair describing the look-up coordinates of our point of interest, it is obvious that the $S^{\prime}(x, y)$ values can be obtained without the need to perform all of the intermediate calculations: only the magnification factor $(M)$ has to be determined.

Our procedure in determining corresponding point pairs from slightly dissimilar objects is based on the assumption of 'local geometrical similarity', applying the above transformation rulc to continuously changing reference point pairs. Alternatively, if two points selected for determining the necessary operations (translation, etc.) are located close together, the calculated transformation rule will be valid for any selected point, the position of which is close enough to the reference point pair, irrespective of the slight differences between the pictures. With this assumption, we have developed an efficient self-teaching algorithm that is well suited to studies where the examiner must continuously trace a reference picture and only occasionally turns to the look-up picture for comparison. The steps of the procedure are:
1. Locate two easily recognizable points near the starting point of the examination. Find the corresponding points on the look-up picture and store the coordinates in the reference database.

2. Begin the examination by finding the first point to be correlated. Determine the 'local' transformation by using the first two point pairs of the reference database. Localize your corresponding field at the predicted position. Correct the predicted coordinates, if necessary, then add the precise coordinates of your newly located reference and look-up points to the database.

3. Continue by finding the next point to be correlated. Determine the closest two reference points from the reference database. Determine the 'local' transformation by using these two points and their corresponding look-up pairs. Localize your area of interest at the predicted place. Correct predicted coordinates, if necessary, then add the precise coordinates of your newly localized reference and look-up points to the database.

4. Repcat from '3.' or finish.

\section{Discussion}

The algorithm described above is an efficient self-teaching procedure for correlating corresponding points on slightly dissimilar objects (pictures). The calculations are based on transformation rules introduced for geometrically similar profiles (affine transforms) and the application of continually changing reference point base which ensures the compensation of small differences in appearance of the objects. Furthermore, there is no need to build up a reference point basis prior to examination, as the database is developed during the examination process. The procedure is especially applicablc to examination strategies aimed at the correlation of selected local features, rather than of whole objects.

Generally, the comparison of two slightly dissimilar images - because of the violation of their geometrical similarity - would require the devel- 
opment of a two-dimensional continuous transformation field for the reference object (see, for example, Ref. 12). In the practical approximation presented this continuous field was substituted with sets of transformation parameters defined at discrete sample points. This procedure implicitly involves the assumption that the transformation defined at a particular point is valid within a non-infinitesimal neighborhood of that point. Naturally, the precision of the actual transformation formula at a selected point decays with the distance measured from that point. The accuracy of the transformation, i.e., the deviation between calculated and true values of the corresponding coordinates, is influenced by (i) the density of the reference points, (ii) size and recognizability of tissue features used as reference points and (iii) the measure of the violation of the geometrical similarity. The density of the reference points can be chosen in such a way that a satisfactory correlation can be obtained in each practical application of the algorithm if the corresponding features on the two images can be unequivocally recognized and localized.

The algorithm presented above itself exhibits the core of the calculation rules for point-to-point correlation of objects with location-dependent transformation parameters. It is the matter for the programmer how the algorithm is coded into a programming language, adapted to a particular application problem or extended with safety devices such as re-tracing, back-tracing or additional database reviewing capabilities. A simplified version of the algorithm has been implemented in our laboratory to an application task where the correlation of ultrastructural features (synapses) on consecutive sections was aimed [14]. The procedure proved to be satisfactory, as the deviation of the predicted positions from their true values was in no case larger than $5 \mu \mathrm{m}$ in situations when the linear dimensions of the objects (used as reference points) were between 0.4 $\mu \mathrm{m}$ and $0.7 \mu \mathrm{m}$, the density of reference points was approximately $3.0 \times 10^{-3} / \mu \mathrm{m}^{2}$ and the distance between positions to be compared was usually larger than $1 \mathrm{~mm}$.

\section{References}

1 L.K. Turner, Y.-C. Ling, M.T. Bernius and G.H. Morrison, Direct correlation of ion and electron microscopic images by digital image superpositioning. Anal. Chem. 59 (1987) $2463-2468$.

2 E. Ito, R. Kudo, M. Miyoshi, S. Tanaka, K. Kumai, T. Takashina and $\mathbf{M}$. Hashimoto, Transmission and scanning electron microscopic study of the same cytologic material, Acta Cytol. 32 (1988) 588-592.

3 H.E. Pattce, S.C. Mohapatra and E.K. Angello, A technique for comparative light and scanning electron microscopy of the same deparaffinized microtomed section, Journal of Microscopy 137 (1985) 111-114.

4 L.R. Swenney and B.L. Shapiro, One to one correlation of histological and histochemical light microscopy with scanning electron microscopy, Scanning Electron Microsc. ? (1981) 63-72.

5 L. Siklós, M. Rózsa and J. Zombori, A simple method for correlative light, scanning electron microscopic and X-ray microanalytical examination of the same section, J. Microsc. 142 (1986) 107-110.

6 K.G. Kupke, J.P. Pickett, P. Ingram, D.P. Griffis, R.W. Linton, P.C. Burger and J.D. Schelburne, Preparation of biological tissue sections for correlative ion, electron and light microscopy, J. Electron Microsc. Technol. 1 (1984) 299-309.

7 M. Moss and V. Howard, Look before you leap, Lab. Pract., 37 (1988) 15-23

8 H.J.G. Gundersen. Quantitative analysis of three-dimensional structures in neuroanatomy. In: Quantitative neuroanatomy in transmitter research, eds. L.F. Agnati and $\mathrm{K}$ Fuxe, pp. 3-9 (MacMillan. London, 1985).

9 H.J.G. Gundersen. P. Bagger, T.F. Bendtsen, S.M. Evans, L. Korbo, N. Marcussen, A. Moller, K. Nielsen, J.R. Nyengaard, B. Pakkenberg, F.B. Sorensen. A. Vesterby and M.J. West. The new stereological tools: Disector. fractionator, nucleator and point sampled intercepts and their use in pathological research and diagnosis, Acta Pathol. Microbiol. Immunol. Scand. 96 (1988) 857-881.

10 D.C. Sterio, The unbiased estimation of number and sizes of arbitrary particles using the disector, J. Microsc. 134 (1984) 127-136.

11 M. Rickmann, L. Siklós, F. Joó and J.R. Wolff, $X$-Y-recording in transmission electron microscopy. A versatile and inexpensive interface to personal computers with application to stereology, J. Microsc. 159 (1990) 289-299.

12 F.L. Bookstein, The measurement of biological shape and shape change. Lecture notes in biomathematics. Vol. 24., ed. S. Levin, Springer Berlin, 1978).

13 R.C. Gonzalez and P. Wintz. Digital image processing. (Addison-Wesley, Reading, 1977).

14 l. Siklós. Á. Párducz, N. Halász, M. Rickmann, F. Joó and J.R. Wolff, An unbiased estimation of the total number of synapses in the superior cervical ganglion of adult rats established by the disector method. Lack of change after long-lasting sodium bromide administration, J. Neurocytol. 19 (1990) 443-454. 